\title{
Sensibilidade a antimicrobianos de bactérias isoladas do trato respiratório de pacientes com infecções respiratórias adquiridas na comunidade: resultados brasileiros do Programa SENTRY de Vigilância de Resistência a Antimicrobianos dos anos de 1997 e 1998
}

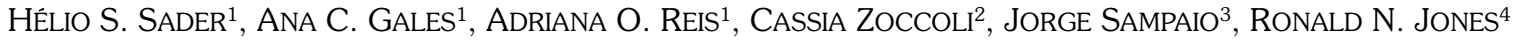

O tratamento da pneumonia adquirida na comunidade (PAC) é habitualmente empírico e o uso de antimicrobianos é baseado em estudos de vigilância. O programa SENTRY foi desenhado para monitorar a resistência a antimicrobianos através de uma rede internacional de laboratórios. Três centros no Brasil participaram do Programa SENTRY em 1997 e em 1998. Métodos: Um total de 344 isolados bacterianos coletados de pacientes com PAC em 1997 e 1998 foram testados contra mais de 20 agentes antimicrobianos pelo método de microdiluição em caldo. Resultados: Entre os $S$. pneumoniae (176 isolados), 71,6\% foram sensiveis à penicilina. Alto nível de resistência à penicilina e resistência à cefotaxima foram encontrados em 2,3 e 4,0\%, respectivamente. As novas quinolonas levofloxacina (MIC90, $2 \mu \mathrm{g} / \mathrm{mL}$ ) e gatifloxacina (MIC90, 0,5 $\mathrm{g} / \mathrm{mL}$ ) foram ativas contra $100 \%$ dos isolados testados. Entre os outros antimicrobianos não $\beta$-lactâmicos testados, os mais ativos foram (\% de sensibilidade): cloranfenicol $(97,5 \%)>$ clindamicina $(94 \%)>$ azitromicina $(90,3 \%)>$ claritromicina

$(89,4 \%)>$ tetraciclina $(76,4 \%)>$ sulfametoxazol/trimetoprim $(60,2 \%)$. A percentagem de

Haemophilus influenzae (101 isolados) resistentes à amoxicilina foi de 90,1\%, enquanto entre Moraxella catarrhalis (67 isolados) somente 9,0\% foram sensiveis. O ácido clavulânico restaurou a atividade de amoxicilina contra $H$. influenzae e $M$. catarrhalis. Porém, $H$. influenzae demonstrou niveis aumentados de resistência para sulfametoxazol/trimetoprim (55,1\% de sensibilidade), claritromicina ( $80,4 \%$ de sensibilidade) e cefaclor $(88,2 \%$ de sensibilidade). Todos os isolados de $H$. influenzae e M. catarrhalis foram sensiveis à levofloxacina (MIC $90, \leq 0,5 \mu \mathrm{g} / \mathrm{mL}$ para ambos) e gatifloxacina (MIC90, $\leq 0,06 \mu \mathrm{g} / \mathrm{mL}$ para ambos) apresentando MICs muito baixos. Conclusões: Os resultados indicam que a prevalência de $S$. pneumoniae com alto grau de resistência à penicilina é ainda baixa no Brasil; porém, a prevalência de $S$. pneumoniae com resistência intermediária à penicilina e resistência cruzada a outras classes de antimicrobianos é relativamente alta em nosso meio. Por outro lado, as novas quinolonas são altamente ativas contra $S$. pneumoniae e outros patógenos responsáveis por infecções respiratórias adquiridas na comunidade.

(J Pneumol 2000;27(1):25-34)

1. Laboratório Especial de Microbiologia Clínica, Disciplina de Doenças Infecciosas e Parasitárias da Universidade Federal de São Paulo, São Paulo, Brasil.

2. Laboratório Médico Santa Luzia, Florianópolis, Brasil.

3. Laboratório Lâmina, Rio de Janeiro, Brasil.

4. Departamento de Patologia da Faculdade de Medicina da Universidade de Iowa, lowa City, Iowa, EUA.
Endereço para correspondência - Prof. Dr. Hélio S. Sader, Laboratório Especial de Microbiologia Clínica, Disciplina de Doenças Infecciosas e Parasitárias, Universidade Federal de São Paulo-EPM, Rua Botucatu, 740 - 04023-900 - São Paulo, SP. Tels. (11) 5081-2819/571-5180/ 576-4393; E-mail: heliosader@uol.com.br

Recebido para publicação em 11/5/00. Reapresentado em 20/ 9/00. Aprovado, após revisão, em 16/10/00. 


\title{
Susceptibility to respiratory tract isolated bacteria to antimicrobial agents in patients with community-acquired respiratory tract infections: 1997 and 1998 Brazilian data of the SENTRY surveillance program of resistance to antimicrobial agents
}

\begin{abstract}
Background: Antimicrobial treatment of community-acquired respiratory tract infections (CARTI) is usually empiric and antibiotics are chosen on the basis of surveillance studies. The SENTRY Program was designed to monitor antimicrobial resistance via a worldwide surveillance network of sentinel laboratories. Three sites in Brazil participated in the 1997 and 1998 SENTRY Program. Methods: A total of 344 bacterial isolates, collected from patients with CARTI in 1997 and 1998, were tested against more than 20 antimicrobial agents by the broth microdilution method. Results: Among S. pneumoniae (176 isolates), $71.6 \%$ were susceptible to penicillin. High level resistance to penicillin and resistance to cefotaxime was found in 2.3 and $4.0 \%$, respectively. The newer quinolones, levofloxacin, (MIC90 of $2 \mu \mathrm{g} / \mathrm{mL}$ ) and gatifloxacin (MIC90 of $0.5 \mu \mathrm{g} / \mathrm{mL}$ ) were active against $100 \%$ of the isolates tested. Among the other non- $\beta$-lactam drugs tested, the rank order of pneumococci activity was (\% susceptible): chloramphenicol $(97.5 \%)>$ clindamycin (94.0\%) > azithromycin (90.3\%) > clarithromycin (89.4\%) > tetracycline (76.4\%) > trimethoprim/ sulfamethoxazole (60.2\%). The percentage of Haemophilus influenzae (101 isolates) susceptible to amoxicillin was $90.1 \%$, whereas among Moraxella catarrhalis (67 isolates) only 9.0\% were susceptible. Clavulanic acid restored the activity of amoxicillin against both $\mathrm{H}$. influenzae and $\mathrm{M}$. catarrhalis. However, $\mathrm{H}$. influenzae showed increased levels of resistance to trimethoprim/ sulfametoxazole (55.1\% susceptibility), clarithromycin (80.4\% susceptibility), and cefaclor (88.2\%) susceptibility). All $\mathrm{H}$. influenzae and $\mathrm{M}$. catarrhalis isolates were susceptible to levofloxacin (MIC90, $\leq 0.5 \mu \mathrm{g} / \mathrm{mL}$ for both) and gatifloxacin (MIC90, $\leq 0.06 \mu \mathrm{g} / \mathrm{mL}$ for both) with very low MICs.

Conclusion: Results indicate that the rate of $\mathrm{S}$. pneumoniae showing high-level penicillin resistance is still low in Brazil. However, intermediate resistance to penicillin associated with resistance to other classes of antimicrobial agents was relatively high. On the other hand, the new quinolones were highly active against $100 \%$ of the respiratory pathogens tested.
\end{abstract}

Descritores - Infecções respiratórias. Pneumonia bacteriana. Resistência microbiana a drogas. Avaliações de programas. Vigilância de resistência. Teste de sensibilidade.

Key words - Respiratory tract infections. Bacterial pneumonia. Microbial drug resistance. Program evaluation. Microbial sensibility tests.

\section{INTRODUÇÃO}

As infecções respiratórias representam uma causa importante de morbidade e mortalidade, sendo a pneumonia a causa infecciosa mais freqüente de morte nos Estados Unidos ${ }^{(1)}$. A pneumonia adquirida na comunidade (PAC) acomete mais de 4 milhões de pessoas anualmente e a mortalidade depende da gravidade do quadro. Pacientes que não são internados apresentam taxas de mortalidade inferiores a $5 \%$, enquanto aqueles que são internados, $e$ que representam 20 a $25 \%$ dos com PAC, apresentam taxas de mortalidade de 12 a $25 \%(2)$.

O diagnóstico clínico de pneumonia não apresenta dificuldade. A dificuldade maior consiste em relacionar o
Siglas e abreviaturas utilizadas neste trabalho

PAC - Pneumonia adquirida na comunidade

diagnóstico sindrômico da pneumonia aos diferentes agentes etiológicos que podem causá-la. Para isso devemos utilizar dados epidemiológicos, radiológicos e clínicos. São raros os estudos brasileiros que avaliam os agentes etiológicos mais freqüentes; porém, vários estudos internacionais mostram que as bactérias "típicas" que mais freqüentemente causam pneumonias comunitárias são Streptococcus pneumoniae e Haemophilus influenzae ${ }^{(3-5)}$. Na verdade, esses são os principais agentes etiológicos de outros tipos de infecções respiratórias, tais como sinusite, otite média e exacerbação aguda de bronquite crônica. Além disso, alguns estudos mostram que a Moraxella catarrhalis e o Staphylococcus aureus também representam causas freqüentes de infecções respiratórias ${ }^{(4)}$.

Além das bactérias citadas acima, as pneumonias adquiridas na comunidade podem também ser causadas pelas 
chamadas bactérias "atípicas", que incluem Mycoplasma pneumoniae, Chlamydia pneumoniae e Legionella pneumophila ${ }^{(3,5)}$. Em um recente estudo brasileiro esses patógenos foram responsáveis por cerca de metade dos casos de PAC nos quais se conseguiu estabelecer o diagnóstico etiológico(6). É importante enfatizar também que a diferenciação clínica entre pneumonias "típicas" e "atípicas" é extremamente difícil ${ }^{(5-7)}$.

Grande número de estudos tem avaliado o perfil de sensibilidade das bactérias que mais freqüentemente causam pneumonias, especialmente $S$. pneumoniae e $H$. influenzae; porém, estudos que avaliam amostras brasileiras ainda são $\operatorname{raros}^{(8-10)}$. Os resultados de programas locais de vigilância de resistência a antimicrobianos são de extrema importância, pois auxiliam a escolha da terapêutica empírica, especialmente em infecções na quais o diagnóstico etiológico é difícil.

O Programa SENTRY de Vigilância de Resistência (SENTRY Antimicrobial Surveillance Program) é um programa mundial e longitudinal de vigilância de resistência a antimicrobianos. Esse programa apresenta as seguintes características principais: a) avalia várias regiões do mundo de forma longitudinal; b) utiliza testes de sensibilidade de referência (microdiluição em caldo) para avaliação das bactérias; c) coleta de bactérias dos importantes sítios de infecções; d) avaliação de grande número de antimicrobianos $^{(8,11,12)}$. A coleta de amostras é feita de acordo com o sítio de infecção e inclui cinco objetivos: a) infecções da corrente sanguínea; b) infecções comunitárias do trato respiratório; c) pneumonia hospitalar; d) infecção de ferida cirúrgica; e) infecções do trato urinário. Apresentamos aqui a avaliação de amostras bacterianas coletadas de pacientes com infecções respiratórias adquiridas na comunidade $e$ atendidos nos centros brasileiros que participaram do Programa SENTRY em 1997 e 1998.

\section{MATERIAL E MÉTODOS}

Amostras bacterianas: As bactérias foram coletadas em três laboratórios de microbiologia, o Laboratório Especial de Microbiologia Clínica da Universidade Federal de São Paulo, o Laboratório Lâmina, do Rio de Janeiro, e o Laboratório Médico Santa Luzia, de Florianópolis, $\mathrm{SC}$.

Foi solicitado que cada laboratório participante enviasse aproximadamente 40 amostras de $S$. pneumoniae, 40 amostras de $H$. influenza e 20 amostras de M. catarrhalis coletadas consecutivamente entre maio e setembro de cada ano (1997 e 1998). Somente uma amostra de cada paciente foi incluída no estudo. Foram incluídas no estudo apenas bactérias isoladas de espécimes clínicos que preenchessem os critérios de aceitabilidade preconizados pela Sociedade Americana de Microbiologia, isto é, baixo número de células epiteliais e alto número de leucócitos, além de correlação com o resultado do exame direto de Gram ${ }^{(13,14)}$.

As bactérias foram identificadas (gênero e espécie) pelo laboratório participante e enviadas para o centro coordenador (Universidade de Iowa, Iowa City, IA, EUA) para avaliação da sensibilidade a antimicrobianos e realização dos demais testes laboratoriais.

Avaliação da sensibilidade a antimicrobianos: Foi realizada pela técnica de microdiluição em caldo conforme descrito pelo National Committee for Clinical Laboratory Standards (NCCLS), que é o órgão responsável pela padronização de técnicas laboratoriais nos $\operatorname{EUA}^{(15,16)}$. Os antimicrobianos foram obtidos dos respectivos fabricantes e incluem a maioria dos antibióticos disponiveis comercialmente. Além disso, vários compostos que ainda se encontravam em fase de investigação durante o período do estudo também foram avaliados. Estes incluem as fluoroquinolonas gatifloxacina (Tequin ${ }^{\circledR}$, Bristol-Myers Squibb), e trovafloxacina (Pfizer Inc.), a estreptogramina quinupristin-dalfopristin (Synercid ${ }^{\circledR}$, Rhone-Poulenc Rorer) e a oxazolidinona linezolid (Pharmacia \& UpJohn).

Controle de qualidade: Todos os testes foram realizados sob rígido controle de qualidade através da avaliação das seguintes cepas controles: Escherichia coli ATCC 25922, Staphylococcus aureus ATCC 29213, Pseudomonas aeruginosa ATCC 27853 e Enterococcus faecalis ATCC 29212. A interpretação dos testes foi realizada de acordo com a padronização recomendada pelo NCCLS ${ }^{(15,16)}$.

\section{RESULTADOS}

Foi coletado um total de 344 amostras bacterianas, sendo 176 amostras de S. pneumoniae (ou pneumococo), 101 amostras de $H$. influenzae e 67 amostras de $M$. catarrhalis. Os resultados da avaliação da sensibilidade desses patógenos a mais de 20 antimicrobianos parenterais e orais são mostrados na Tabela 1 . Além da percentagem de amostras sensiveis, a Tabela 1 apresenta também os valores de MIC50 e MIC90, que representam as concentrações necessárias para inibir $50 \%$ e $90 \%$ das amostras, respectivamente. Esses valores permitem comparar a potência dos antimicrobianos, pois, quanto menor a concentração necessária para inibir a bactéria, mais potente será o antimicrobiano.

Somente pouco mais de $70 \%$ das amostras de pneumococos foram considerados sensiveis à penicilina (MIC $\leq$ $0,06 \mu \mathrm{g} / \mathrm{mL}$ ); a maioria das amostras não-sensiveis apresentou resistência intermediária (MIC, $0,06-1 \mu \mathrm{g} / \mathrm{mL}$ ). Somente 2,3\% das amostras apresentaram alto grau de resistência (MIC $\geq 2 \mu \mathrm{g} / \mathrm{mL}$ ). A amoxicilina e amoxicilina/ clavulanato apresentaram taxas de sensibilidade mais ele- 
vadas que a penicilina devido a diferenças de pontos de corte ou breakpoints, pois a atividade antimicrobiana desses compostos é muito semelhante para o pneumococo. Enquanto que, para ser considerado sensivel à penicilina, o MIC deve ser $\leq 0,06 \mu \mathrm{g} / \mathrm{mL}$, para amoxicilina $e$ amoxicilina/clavulanato o breakpoint é $\leq 2 \mu \mathrm{g} / \mathrm{mL}^{(15)}$. Essa diferença é decorrente do fato de o breakpoint para penicilina considerar os casos de meningite. Devido à baixa penetração liquórica do antimicrobiano, pode ocorrer falha terapêutica em meningites causadas por amostras que apresentem resistência intermediária (MIC, $0,06-1 \mu \mathrm{g} / \mathrm{mL}$ ). Por outro lado, alguns estudos têm mostrado que pneumonias causadas por pneumococos com resistência intermediária aos $\beta$-lactâmicos podem ainda ser tratadas com doses mais elevadas desses antimicrobianos ${ }^{(17)}$.

Com relação aos antibióticos $\beta$-lactâmicos, amostras de pneumococos sensiveis à penicilina devem ser consideradas sensiveis também à ampicilina, amoxicilina, amoxicilina/clavulanato, ampicilina/sulbactam, cefaclor, cefdinir, cefepime, cefetamet, cefixime, cefotaxime, cefprozil, ceftibuten, ceftriaxone, cefuroxime, cefpodoxime, ceftizoxime, imipenem, loracarbef e meropenem. Amostras não-sensiveis à penicilina ( $\mathrm{MIC}>0,06 \mu \mathrm{g} / \mathrm{mL}$ ) poderão ser testadas para cefuroxime, ceftriaxona, cefotaxime, imipenem ou meropenem, pois há definição de breakpoints (somente MIC) para esses compostos. Não se recomenda o uso dos demais $\beta$-lactâmicos citados para o tratamento de qualquer tipo de infecção causada por amostras de pneumococo não-sensiveis à penicilina ${ }^{(15)}$. Outros $\beta$-lactâmicos não citados, como cefalexina por exemplo, apresentam atividade baixa mesmo contra amostras de pneumococo sensiveis à penicilina.

A atividade dos antimicrobianos contra os pneumococos foi avaliada também de acordo com a sensibilidade destes à penicilina (Tabela 2). Verificou-se que, conforme o pneumococo se torna mais resistente à penicilina, as taxas de resistência aumentam para outros $\beta$-lactâmicos e mesmo para antimicrobianos de outras classes, como macrolídeos e sulfametoxazol/trimetoprim. Os macrolídeos claritromicina e azitromicina foram ativos contra quase $95 \%$ dos pneumococos sensiveis à penicilina, mas inibiram apenas 78 a $80 \%$ dos pneumococcos não-sensiveis à penicilina. O sulfametoxazol/trimetoprim apresentou baixa atividade mesmo contra pneumococos de ma-

TABELA 1

Atividade in vitro de 23 antibióticos parenterais e orais contra Streptococcus pneumoniae, Haemophilus influenzae e Moraxella catarrhalis

\begin{tabular}{|c|c|c|c|c|c|c|}
\hline \multirow[b]{2}{*}{ Antibiótico } & \multicolumn{2}{|c|}{ H. influenzae (101) } & \multicolumn{2}{|c|}{ M. catarrhalis (67) } & \multicolumn{2}{|c|}{ S. pneumoniae (176) } \\
\hline & $\mathrm{MIC}_{50 / 90}$ & 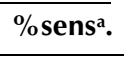 & $\mathrm{MIC}_{50 / 90}$ & $\overline{\% \text { sens. }^{\mathrm{a}}}$ & $\mathrm{MIC}_{50 / 90}$ & \%sens. ${ }^{a}$ \\
\hline Amoxicilina & $0,5 / 4$ & 90,1 & $2 / 4$ & 9,0 & $\leq 0,06 / 0,12$ & 97,5 \\
\hline Penicilina & $0,25 / 2$ & 90,1 & $4 / 8$ & 9,0 & $0,03 / 0,25$ & $71,6(2,3)^{b}$ \\
\hline Amoxicilina/clavulanato & $0,5 / 0,5$ & 100,0 & $0,25 / 0,25$ & 98,5 & $0,25 / 0,25$ & 97,5 \\
\hline Cefaclor & $4 / 16$ & 88,2 & $0,5 / 2$ & 100,0 & $1 / 2$ & $71,6^{\mathrm{a}}$ \\
\hline Cefuroxima & $1 / 2$ & 96,3 & $1 / 2$ & 100,0 & $\leq 0,06 / 0,5$ & 94,0 \\
\hline Cefprozil & $2 / 8$ & 93,5 & $2 / 8$ & 95,5 & $0,25 / 2$ & $71,6^{\mathrm{a}}$ \\
\hline Cefixima & $0,06 / 0,5$ & 100,0 & $0,25 / 0,5$ & 100,0 & $0,5 / 4$ & $71,6^{\mathrm{a}}$ \\
\hline Cefpodoxima & $0,06 / 0,12$ & 100,0 & $0,5 / 1$ & 100,0 & $\leq 0,03 / 0,25$ & $71,6^{\mathrm{a}}$ \\
\hline Ceftriaxona/cefotaxima & $0,015 / 0,03$ & 100,0 & $0,25 / 0,5$ & 100,0 & $0,03 / 0,12$ & 95,6 \\
\hline Cefepima & $\leq 0,06 / 0,12$ & 100,0 & $0,5 / 2$ & 100,0 & $\leq 0,06 / 0,25$ & 96,0 \\
\hline Azitromicina & $1 / 2$ & 99,1 & $\leq 0,12 / \leq 0,12$ & 100,0 & $\leq 0,12 / 0,5$ & 90,5 \\
\hline Claritromicina & $8 / 16$ & 80,4 & $\leq 0,25 / \leq 0,25$ & 100,0 & $\leq 0,25 / 0,5$ & 89,4 \\
\hline Clindamicina & - & - & - & - & $\leq 0,06 / 0,12$ & 94,0 \\
\hline Ciprofloxacina & $\leq 0,015 / \leq 0,015$ & 100,0 & $0,03 / 0,06$ & 100,0 & $1 / 2$ & - \\
\hline Gatifloxacina & $\leq 0,03 / \leq 0,03$ & 100,0 & $\leq 0,03 / 0,06$ & 100,0 & $0,25 / 0,5$ & 100,0 \\
\hline Trovafloxacina & $\leq 0,03 / \leq 0,03$ & 100,0 & $\leq 0,03 / 0,06$ & 100,0 & $0,25 / 0,5$ & 99,5 \\
\hline Grepafloxacina & $\leq 0,12 / \leq 0,12$ & 100,0 & $\leq 0,12 / \leq 0,12$ & 100,0 & $0,25 / 0,25$ & 100,0 \\
\hline Esparfloxacina & $\leq 0,12 / \leq 0,12$ & 100,0 & $\leq 0,12 / \leq 0,12$ & 100,0 & $0,25 / 0,5$ & 99,0 \\
\hline Levofloxacina & $\leq 0,5 / \leq 0,5$ & 100,0 & $\leq 0,5 / \leq 0,5$ & 100,0 & $1 / 2$ & 100,0 \\
\hline Cloranfenicol & $\leq 2 / \leq 2$ & 95,3 & $\leq 2 / \leq 2$ & 100,0 & $\leq 2 / 4$ & 97,5 \\
\hline Tetraciclina & $\leq 2 / \leq 2$ & 95,3 & $\leq 2 / \leq 2$ & 100,0 & $\leq 2 />16$ & 76,4 \\
\hline Trimetoprim/sulfametoxazol & $0,5 />8$ & 55,1 & $\leq 0,25 / 2$ & 88,2 & $0,5 / 4$ & 60,2 \\
\hline Vancomicina & - & - & - & - & $0,25 / 0,5$ & 100,0 \\
\hline
\end{tabular}

a. Percentagem de sensibilidade determinada de acordo com a teste para penicilina [NCCLS 2000].

b. Percentagem de amostras com alto grau de resistência (MIC $\geq 2 \mu \mathrm{g} / \mathrm{mL}$ ) 
neira geral, inibindo apenas $66,7 \%$ das amostras sensíveis à penicilina e menos da metade (44\%) das amostras não-sensiveis à penicilina.

Por outro lado, as novas quinolonas apresentaram excelente atividade contra pneumococo, independente de sua sensibilidade à penicilina (Tabela 2). Apesar de as taxas de sensibilidade serem bastante altas para todas as quinolonas (exceto para ciprofloxacina, pois não estão definidos breakpoints para esse composto), notamos uma importante diferença de potência quando analisamos os valores de MIC50 e MIC90. Enquanto que para levofloxacina os valores de MIC50 e MIC90 foram, respectivamente, de 1 e $2 \mu \mathrm{g} / \mathrm{mL}$, para as quinolonas mais novas (gatifloxacina, trovafloxacina, grepafloxacina e esparfloxacina) esses valores foram quatro vezes mais baixos, ou seja, 0,25 e $0,5 \mu \mathrm{g} / \mathrm{mL}$ respectivamente (Tabelas 2 e 3 ).

A atividade das quinolonas pode ainda ser comparada utilizando o parâmetro "área abaixo da curva dividido pelo MIC", ou seja, AUC/MIC (area under the curve/minimum inhibitory concentration) (Figura 1). Quanto mais alto esse valor, maior seria a potência da quinolona e maior a probabilidade de uma evolução clínica favorável ${ }^{(18)}$. A Tabela 3 apresenta os resultados do parâmetro AUC/MIC utilizando os valores de AUC relatados na literatura $e$ os valores de MIC90 encontrados em nosso estudo. Como podemos verificar, baseada nesse parâmetro a gatifloxa-

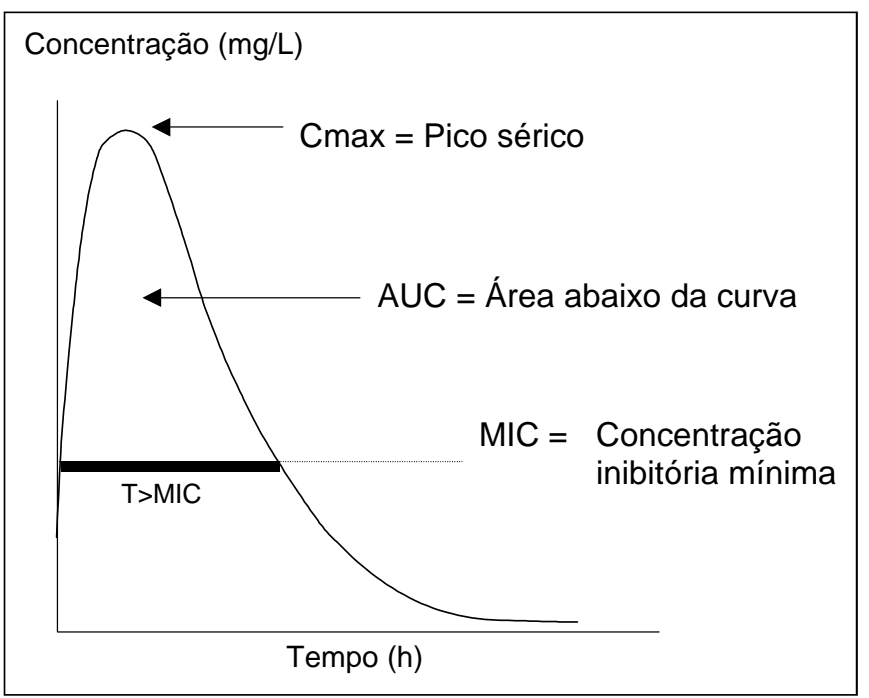

Figura 1 - Parâmetros de farmacocinética-farmacodinâmica relacionados com evolução clínica

TABELA 2

Atividade in vitro de 23 antibióticos parenterais e orais contra 176 amostras de Streptococcus pneumoniae de acordo com a sensibilidade à penicilina

\begin{tabular}{|c|c|c|c|c|}
\hline \multirow[t]{2}{*}{ Antibiótico } & \multicolumn{2}{|c|}{ Sensível à penicilina (126) } & \multicolumn{2}{|c|}{ Não-sensível à penicilina $(50)^{a}$} \\
\hline & $\mathrm{MIC}_{50 / 90}$ & $\%$ susc. & $\mathrm{MIC}_{50 / 90}$ & $\%$ susc. \\
\hline Amoxicilina & $\leq 0,06 / \leq 0,06$ & 100,0 & $\leq 0,06 / 0,5$ & 90,0 \\
\hline Penicilina & $\leq 0,03 / 0,06$ & 100,0 & $0,12 / 1$ & 0,0 \\
\hline Amoxicilina/clavulanato & $0,12 / 0,12$ & 100,0 & $0,12 / 2$ & 90,0 \\
\hline Cefaclor & $0,5 / 1$ & 100,0 & - & - \\
\hline Cefuroxima & $\leq 0,06 / 0,12$ & 98,4 & $0,25 / 2$ & 80,0 \\
\hline Cefprozil & $0,25 / 0,5$ & 100,0 & - & - \\
\hline Cefixima & $0,25 / 0,5$ & 100,0 & - & - \\
\hline Cefpodoxima & $\leq 0,03 / 0,06$ & 100,0 & - & - \\
\hline Cefotaxima/ceftriaxona & $0,015 / 0,03$ & 98,4 & $0,06 / 0,5$ & 90,0 \\
\hline Cefepima & $\leq 0,06 / \leq 0,06$ & 97,6 & $0,12 / 0,5$ & 90,0 \\
\hline Azitromicina & $\leq 0,12 / \leq 0,12$ & 94,4 & $\leq 0,12 / 2$ & 80,0 \\
\hline Claritromicina & $\leq 0,25 / \leq 0,25$ & 94,4 & $\leq 0,25 / 1$ & 78,0 \\
\hline Clindamicina & $\leq 0,06 / 0,12$ & 97,6 & $\leq 0,06 / 4$ & 86,0 \\
\hline Ciprofloxacina & $1 / 2$ & - & $1 / 2$ & - \\
\hline Gatifloxacin & $0,25 / 0,5$ & 100,0 & $0,25 / 0,5$ & 100,0 \\
\hline Trovafloxacina & $0,25 / 0,5$ & 100,0 & $0,25 / 0,5$ & 98,0 \\
\hline Grepafloxacina & $0,25 / 0,25$ & 100,0 & $0,25 / 0,5$ & 100,0 \\
\hline Esparfloxacina & $0,25 / 0,5$ & 98,1 & $0,25 / 0,5$ & 100,0 \\
\hline Levofloxacina & $1 / 2$ & 100,0 & $1 / 2$ & 100,0 \\
\hline Cloranfenicol & $\leq 2 / 4$ & 97,6 & $\leq 2 / 4$ & 96,0 \\
\hline Tetraciclina & $\leq \overline{2} />16$ & 77,0 & $\leq 2 />16$ & 80,0 \\
\hline Trimetoprim/sulfametoxazol & $\leq 0,25 / 4$ & 66,7 & $2 / 8$ & 44,0 \\
\hline Vancomicina & $\overline{0}, 25 / 0,5$ & 100,0 & $0,25 / 0,5$ & 100,0 \\
\hline
\end{tabular}

a. Incluem amostras com resistência intermediária (MIC $0,12-1 \mu \mathrm{g} / \mathrm{mL}$ ) e com alto grau de resistência (MIC $\geq 2 \mu \mathrm{g} / \mathrm{mL}$ ). 
TABELA 3

Atividade in vitro das novas quinolonas e avaliação da atividade de acordo com o parâmetro área sob a curva (AUC) dividido pelo MIC90 encontrado na coleção de bactérias avaliadas

\begin{tabular}{lcccc}
\hline Quinolonas & MIC50 $(\boldsymbol{\mu g} / \mathbf{m L})$ & MIC90 $(\boldsymbol{\mu g} / \mathbf{m L})$ & \% sens $\mathbf{~}^{\mathbf{}}$ & AUC/MIC90 \\
\hline Gatifloxacina & 0,25 & 0,5 & 100,0 & 102,0 \\
Trovafloxacina & 0,25 & 0,5 & 99,4 & 48,8 \\
Grepafloxacina & 0,25 & 0,25 & 100,0 & - \\
Esparfloxacina & 0,25 & 0,5 & 98,8 & - \\
Levofloxacina & 1 & 2 & 100,0 & 24,0 \\
Ciprofloxacina & 1 & 2 & - & 5,8 \\
\hline
\end{tabular}

a De acordo com padronização do NCCLS [2000]. Critério de sensibilidade para gatifloxacina foi definido como $\leq 2 \mu \mathrm{g} / \mathrm{mL}$.

cina foi a quinolona que apresentou maior atividade contra pneumococo.

Cerca de $10 \%$ das amostras de $H$. influenzae avaliadas mostraram-se resistentes à penicilina e amoxicilina. A amoxicilina/clavulanato foi ativa contra $100 \%$ das amostras de $H$. influenzae, indicando que, diferente do que acontece com o pneumococo, a resistência à penicilina $e$ amoxicilina é decorrente da produção de $\beta$-lactamases que são inibidas por clavulanato e outros inibidores de $\beta$ lactamases, como sulbactam e tazobactam. Essas $\beta$-lactamases também conferem certo grau de resistência às cefalosporinas, especialmente aquelas menos estáveis à hidrólise, como cefalexina e cefaclor, por exemplo (Tabela 1). Com relação aos macrolídeos, a azitromicina apresentou maior atividade que a claritromicina contra amostras de $H$. influenzae. O sulfametoxazol/trimetoprim apresentou baixa atividade também contra $H$. influenzae, inibindo apenas pouco mais da metade das amostras testadas $(55,1 \%$ de sensibilidade). Por outro lado, as quinolonas apresentaram excelente atividade contra $H$. influenzae, com MICs bastante baixos e $100 \%$ de sensibilidade.

As amostras de $M$. catarrhalis foram amplamente sensíveis à maioria dos antimicrobianos avaliados, com exceção apenas da penicilina, amoxicilina e sulfametoxazol/ trimetoprim. Mais de $90 \%$ das amostras avaliadas foram resistentes à penicilina e amoxicilina, enquanto sulfametoxazol/trimetoprim foi ativo contra apenas $88,2 \%$ das amostras (Tabela 1).

\section{DISCUSSÃO}

Nas últimas décadas tem-se verificado um progressivo aumento mundial das taxas de resistência bacteriana em patógenos responsáveis por infecções respiratórias, especialmente $S$. pneumoniae, $H$. influenzae e $M$. catarrhalis ${ }^{(8,19-28)}$. Resistência a antimicrobianos nesses patógenos tem sido amplamente reportada na literatura mé- dica. Produção de $\beta$-lactamases em $H$. influenzae e $M$. catarrhalis tornou-se clinicamente importante e o progressivo aumento da resistência do pneumococo aos $\beta$ lactâmicos tem comprometido a eficácia dessa classe de antimicrobianos no tratamento de infecções respiratórias adquiridas na comunidade.

O pneumococo é uma causa freqüente de pneumonia, otite média, sinusites, bronquites e meningites. Estima-se que meio milhão de casos de pneumonia pneumocócica ocorra por ano nos Estados Unidos ${ }^{(29)}$. O pneumococo é o agente etiológico mais freqüente em pneumonias adquiridas na comunidade em praticamente todos os estudos. Dessa maneira, qualquer regime antimicrobiano proposto para esse tipo de infecção deve proporcionar cobertura para esse patógeno. Devido ao rápido aumento de resistência antimicrobiana desse patógeno ocorrido nos últimos anos, a terapêutica empírica de pneumonias deve basear-se nos padrões regionais de sensibilidade dos patógenos mais freqüentes ${ }^{(19)}$.

As taxas de resistência do pneumococo à penicilina $e$ outros $\beta$-lactâmicos encontradas no presente estudo foram semelhantes àquelas relatadas em outros estudos brasileiros $^{(9,10)}$. Apesar de as taxas brasileiras estarem entre as mais baixas relatadas no mundo, essas taxas têm aumentado rapidamente nos últimos anos. Dados do projeto SIREVA mostram que a percentagem de pneumococos não-sensiveis à penicilina (MIC $>0,06 \mu \mathrm{g} / \mathrm{mL}$ ) subiu de menos de 10\% em 1993 para mais de $20 \%$ em 1996(9,30). Além disso, estudos epidemiológicos utilizando técnicas moleculares de tipagem mostram que o aumento da resistência do pneumococo à penicilina está muitas vezes relacionado à disseminação de clones de resistência que se propagaram por vários países do mundo(24). Acreditase que alguns clones apresentam maior facilidade de disseminação, como o clone espanhol/americano (sorotipo 23F) e o clone francês/espanhol (sorotipo 9/14). Dados do projeto SIREVA mostram que esses clones multirresistentes são raros no Brasil e que possuímos grande varie- 
dade de clones com resistência intermediária(24). Dessa maneira, a penetração desses clones multirresistentes em nosso país poderia provocar rápido aumento das taxas de resistência.

A atividade de outros $\beta$-lactâmicos, incluindo amoxicilina, amoxicilina/clavulanato e sete cefalosporinas, avaliados através de MICs ou percentagens de cepas resistentes, variou de acordo com a sensibilidade à penicilina (Tabela 2). Essa tendência tem sido reportada pelo Programa SENTRY em outras partes do mundo(8). É importante ressaltar mais uma vez a discrepância entre as percentagens de sensibilidade para penicilina $(71,6 \%)$ e amoxicilina $(97,5 \%)$. Esses dois compostos possuem atividade antipneumocócica e características farmacológicas muito semelhantes e essa diferença é decorrente somente da diferença de breakpoints. Dessa maneira, somente as amostras com alto grau de resistência à penicilina (MIC, $\geq 2 \mu \mathrm{g} /$ $\mathrm{mL}$ ) apresentaram resistência à amoxicilina (MIC, $\geq 2 \mu \mathrm{g} /$ $\mathrm{mL})^{(15)}$. Como a resistência à penicilina/amoxicilina em pneumococo é decorrente de alteração de sítio de ação modificação das proteínas ligadoras de penicilina ou "PBPs" e não da produção de $\beta$-lactamases, a associação desses $\beta$-lactâmicos com inibidores de $\beta$-lactamases não altera em nada a atividade do $\beta$-lactâmico. Dessa maneira, a atividade da amoxicilina/clavulanato é semelhante à atividade da amoxicilina isoladamente contra pneumococo ${ }^{(8)}$.

Por outro lado, algumas cefalosporinas podem apresentar menor atividade que penicilina ou amoxicilina em decorrência de menor afinidade das PBPs bacterianas. Para muitas cefalosporinas não há definição de breakpoints, não permitindo a categorização do pneumococo em sensível, intermediário ou resistente ${ }^{(15)}$. Porém, a avaliação dos valores de MIC50 e MIC90 permite uma classificação das cefalosporinas em termos de potência antipneumocócica. Das cefalosporinas avaliadas, os menores valores de MIC50/MIC90 foram encontrados para as cefalosporinas de uso endovenoso cefotaxima/ceftriaxona (MIC90, $0,03 \mu \mathrm{g} / \mathrm{mL}$ ) e cefepima (MIC $90,0,12 \mu \mathrm{g} / \mathrm{mL}$ ). Com relação às cefalosporinas orais, o composto mais potente foi a cefpodoxima (MIC90, 0,12 $\mu \mathrm{g} / \mathrm{mL}$ ). Apesar de ter apresentado boa atividade in vitro neste estudo, avaliações que integram atividade in vitro com propriedades farmacocinéticas/farmacodinâmicas mostram que a percentagem de amostras de pneumococos inibidas pelas concentrações de cefixima normalmente alcançadas nos sítios respiratórios é bastante baixa ${ }^{(20,31)}$. Dessa maneira, podemos dizer que as cefalosporinas mais ativas contra pneumococos são: cefpodoxima (MIC50, 0,015 $\mu \mathrm{g} / \mathrm{mL}$ ) > cefuroxima (MIC50, $1 \mu \mathrm{g} / \mathrm{mL}$ ) > cefprozil (MIC50, $2 \mu \mathrm{g} / \mathrm{mL}$ ). Apesar de muito utilizados no Brasil para o tratamento de infecções respiratórias, o cefaclor (MIC50, $4 \mu \mathrm{g} / \mathrm{mL}$ ) e a cefalexina (não avaliada no presente estudo) representam as cefalosporinas com menor atividade antipneumo- cócica. Esses resultados são semelhantes àqueles encontrados em outros estudos ${ }^{(8,20,31)}$. É importante ressaltar também que em alguns sítios, como ouvido médio, por exemplo, a penetração da penicilina/amoxicilina é superior àquela apresentada pelas cefalosporinas. Dessa maneira, a ocorrência de falência terapêutica pode ser alta quando se utilizam cefalosporinas para o tratamento de otite média, mesmo para infecções causadas por amostras com resistência intermediáriaa ${ }^{(32)}$.

Conforme outros estudos também têm mostrado, a sensibilidade aos macrolídeos tende a acompanhar a resistência aos $\beta$-lactâmicos e não há muita diferença entre a atividade in vitro dos compostos dessa classe. Na verdade, o órgão norte-americano responsável pela padronização dos testes de sensibilidade (National Committee for Clinical Laboratory Standards [NCCLS]) indica que a sensibilidade ou resistência à azitromicina, claritromicina e diritromicina pode ser avaliada testando-se somente a eritromicina. Dessa maneira, amostras resistentes à eritromicina devem ser consideradas resistentes aos demais macrolídeos ${ }^{(15)}$.

A baixa atividade do sulfametoxazol/trimetoprim contra amostras brasileiras de pneumococos deve estar relacionada ao amplo uso desse antimicrobiano em nosso meio. A tetraciclina também apresentou atividade relativamente baixa, mesmo contra amostras sensiveis à penicilina ( $77 \%$ de sensibilidade). Por outro lado, o cloranfenicol apresentou excelente atividade contra pneumococos com mais de $95 \%$ de sensibilidade, mesmo contra cepas resistentes à penicilina. O uso desse antimicrobiano é limitado pelo relato, embora infreqüente, de casos graves de toxicidade hematológica. Porém, o cloranfenicol pode representar boa opção terapêutica em regiões onde os recursos são escassos.

As novas quinolonas apresentaram excelente atividade antipneumocócica, independentemente da sensibilidade à penicilina. Esses novos compostos, especialmente o grupo das 8-metoxi-quinolonas, que inclui gatifloxacina, trovafloxacina e moxifloxacina, apresentam maior potência contra bactérias Gram-positivas, especialmente pneumococos, quando comparadas com quinolonas mais antigas ou mesmo levofloxacina ${ }^{(33-35)}$. A superioridade desses novos compostos pode ser verificada tanto pela avaliação dos MICs quanto pela avaliação destes em associação com características farmacocinéticas/farmacodinâmicas, ou seja, AUC/MIC90 (Tabela 3). A moxifloxacina, que não foi avaliada nesse estudo, apresenta atividade muito semelhante à da gatifloxacina, com MIC90 ao redor de $0,25 \mu \mathrm{g} /$ $\mathrm{mL}$ e AUC/MIC90 ao redor de $120 \mathrm{mg} \mathrm{h} / \mathrm{L}^{(35-37)}$.

Com relação ao $H$. influenzae, verificamos uma taxa de resistência à penicilina/amoxicilina relativamente baixa quando comparada com a de outros países ${ }^{(27)}$. Enquanto, nos Estados Unidos, cerca de um terço das amostras 
de H. influenzae é resistente à amoxicilina em decorrência da produção de $\beta$-lactamases, no Brasil a percentagem de cepas produtoras de $\beta$-lactamases é de cerca de 10 a $15 \%(10,21,27)$. As cefalosporinas de primeira geração $e$ muitas cefalosporinas de segunda geração apresentam atividade reduzida contra amostras produtoras de $\beta$-lactamases; porém, cefuroxima e as cefalosporinas parenterais e orais de terceira geração apresentaram excelente atividade contra esses patógenos (Tabela 1$)^{(27)}$.

Amostras de H. influenzae geralmente apresentam MICs elevados para os macrolídeos, sendo o macrolídeo mais potente contra esse patógenos a azitromicina. Porém, apesar de apresentar altas taxas de sensibilidade para esse antimicrobiano $(99,1 \%)$, podemos verificar que o MIC do $H$. influenzae para a azitromicina (MIC90,2 $2 \mu \mathrm{g} / \mathrm{mL}$ ) é muito superior àqueles encontrados para os $\beta$-lactâmicos mais potentes e para as quinolonas (Tabela 1). Da mesma forma que contra pneumococos, o sulfametoxazol/trimetoprim apresentou baixa atividade contra $H$. influenzae (55,1\% de sensibilidade), devendo então ser evitado seu uso no tratamento de infecções respiratórias. O cloranfenicol e a tetraciclina, ambos com 95,3\% de sensibilidade, apresentaram boa atividade contra $H$. influenzae no presente estudo (Tabela 1).

Conforme verificado em outros estudos, a grande maioria das amostras de $M$. catarrhalis $(91,0 \%)$ foi resistente à penicilina/amoxicilina devido à produção de $\beta$-lactamases. Com exceção do sulfametoxazol/trimetoprim $(88,2 \%$ de sensibilidade), todos os outros antimicrobianos avaliados foram ativos contra mais de $95 \%$ das amostras testadas. Apesar de o isolamento desse agente ser relativamente raro em nosso meio, vários levantamentos colocam a $M$. catarrhalis como uma importante causa de pneumonia e outras infecções respiratórias ${ }^{(6,27)}$. Além disso, não devemos descartar a possibilidade de a baixa freqüência desse patógeno em nosso meio estar relacionada a problemas técnico-laboratoriais.

Em geral, podemos dizer que as opções terapêuticas para o tratamento de infecções respiratórias adquiridas na comunidade incluem várias classes de antimicrobianos, sendo as principais os $\beta$-lactâmicos, macrolídeos, tetraciclinas, sulfas e quinolonas ${ }^{(1,3,5,7,26)}$. As altas taxas de resistência do $S$. pneumoniae e $H$. influenzae ao sulfametoxazol/trimetoprim e do $S$. pneumoniae à tetraciclina encontradas nesse estudo mostram que esses antimicrobianos não representam opções terapêuticas adequadas em nosso meio. Com relação aos $\beta$-lactâmicos, além das taxas crescentes de resistência do $S$. pneumoniae e do $H$. influenzae, esses antimicrobianos não apresentam atividade adequada contra as chamadas bactérias "atípicas", que representam importante causa de pneumonia adquirida na comunidade ${ }^{(6,32)}$. Os resultados desse $e$ de outros estudos brasileiros mostram também que os macrolídeos estão perdendo atividade contra $S$. pneumoniae e $H$. influenzae em nosso meio ${ }^{(10,32)}$. Por outro lado, as novas quinolonas apresentaram excelente atividade contra patógenos avaliados nesse estudo, independente da sensibilidade destes a outras classes de antimicrobianos. Esses resultados corroboram outros estudos brasileiros e internacionais, indicando que esses novos antimicrobianos trazem uma grande contribuição para o tratamento de infecções respiratórias adquiridas na comunidade $(8,27,34,36-38)$.

Dentre quinolonas indicadas para tratamento de infecções respiratórias avaliadas nesse estudo, somente a levofloxacina e a gatifloxacina estão disponiveis comercialmente no Brasil. Além dessas duas, temos também a moxifloxacina, que foi aprovada para uso clínico recentemente no Brasil(37). Além da potência e eficácia contra os prováveis patógenos responsáveis pela infecção, a escolha do antimicrobiano deve levar em consideração também uma série de outros fatores, como, por exemplo, efeitos colaterais, comodidade posológica e custo ${ }^{(39)}$. Os resultados deste e de outros estudos mostram que a gatifloxacina apresenta atividade in vitro superior à levofloxacina. Vários estudos têm demonstrado também que tanto a levofloxacina quanto a gatifloxacina são quinolonas bastante seguras; porém, há descrição de prolongamento do intervalo QT no eletrocardiograma em decorrência do uso de moxifloxacina e outras quinolonas já retiradas do mercado, sendo necessária maior experiência clínica com esses compostos para o melhor conhecimento de possiveis efeitos colaterais ${ }^{(39,40)}$. Apesar de as novas quinolonas representarem atraentes opções terapêuticas para o tratamento de infecções respiratórias adquiridas na comunidade devido à alta potência e espectro antimicrobiano associados a outras características citadas anteriormente, outras classes de antimicrobianos, especialmente $\beta$ lactâmicos e macrolídeos, ainda permanecem como importantes opções terapêuticas em muitas situações clínicas.

\section{AgRADECIMENTOS}

O autores agradecem a Eliete Miranda, Rodrigo E. Mendes, Richard Hollis, Kari Kluger P. Winokur pela importante contribuição na realização dos testes laboratoriais e análise dos resultados, e a todas as pessoas que contribuíram com a coleta das amostras e dos dados epidemiológicos em cada laboratório participante. O Programa SENTRY de Vigilância de Resistência é inteiramente patrocinado pela Bristol Myers Squibb Company.

\section{REFERÊNCIAS}

1. Niederman MS, Bass JB, Campbell GD, et al. Guidelines for the initial management of adults with community-acquired pneumonia: diagnosis, assessment of severity, and initial antimicrobial therapy. Am Rev Respir Dis 1993;148:1418-1426. 
2. Fine MJ, Smith MS, Carson CA, et al. Prognosis and outcome of patients with community-acquired pneumonia: a meta-analysis. JAMA 1996;275:134-141.

3. Ball P. Community-acquired pneumonia: etiology, epidemiology and treatment. Chest 1995;108:355-425.

4. Goldestein F, Bryskier A, Appelbaum PC, Bauernfeind A, Schito GC, Wise R. The etiology of respiratory tract infections and antibacterial activity of fluoroquinolones and other oral bacterial agents against respiratory pathogens. Clin Microbiol Infect 1998;4(Suppl 2):S8-18.

5. Nakatani J, Holanda MA. Pneumonias adquiridas na comunidade e no hospital. In: Prado FC, Ramos J, Valle JR, eds. Atualização terapêutica. São Paulo: Artes Médicas, 1999

6. Rocha RT, Vital AC, Silva COS, Pereira CAC, Nakatani J. Pneumonia adquirida na comunidade em pacientes tratados ambulatorialmente: aspectos epidemiológicos, clínicos e radiológicos das pneumonias atípicas e não atípicas. J Pneumol 2000;1:5-14.

7. Barlett JG, Breiman RF, Mandell LA, File Jr TM. Community-acquired pneumonia in adults: guidelines for management. Clin Infect Dis 1998; 26:811-838.

8. Doern GV, Pfaller MA, Kugler K, Freeman J, Jones RN. Prevalence of antimicrobial resistance among respiratory tract isolates of Streptococcus pneumoniae in North America: 1997 results from the SENTRY antimicrobial surveillance program. Clin Infect Dis 1998;27:764770 .

9. Brandileone MCC, Vieira VSD, Casagrande ST, et al. Prevalence of sorotypes and antimicrobial resistance of Streptococcus pneumoniae strains isolated from Brazilian children with invasive infections. Microb Drug Resist 1997;3:141-146.

10. Critchley IA, Thornsberry C, Hickey ML, et al. Antimicrobial susceptibility of Streptococcus pneumoniae, Haemophilus influenzae and Moraxella catarrhalis collected from five centers in Brazil, 1998. Clin Microbiol Infect 2000;6:178-184.

11. Pfaller MA, Jones RN, Doern GV, Krugler K and the Sentry Participants Group. Bacterial pathogens isolated from patients with blood stream infection: frequencies of occurrence and antimicrobial susceptibility patterns from SENTRY Antimicrobial Surveillance Program (United States and Canada, 1997). Antimicrob Agents Chemother 1998;42: 1762-1770.

12. Sader HS, Jones RN, Gales AC, Klugler K, Pfaller MA, Doern GV, and the SENTRY Latin America Study Group. Antimicrobial susceptibility of patterns for pathogens isolated from patients in Latin American medical centers with a diagnosis of pneumonia: Results from the SENTRY Antimicrobial Surveillance Program (1997). Diagn Microbiol Infect Dis 1998;32:289-301.

13. Reimer LG, Carroll KC. Role of the microbiology laboratory in the diagnosis of lower respiratory tract infections. Clin Infect Dis 1998; 26:742-748.

14. Sneed JO. Processing and interpretation of respiratory tract specimens. In: Isenberg HD, ed. Clinical microbiology procedures handbook. Washington: American Society of Microbiology, 1992;1:15.18.

15. National Committee for Clinical Laboratory Standards. Performance standards for antimicrobial susceptibility testing; tenth informational supplement M100-S10. Wayne, PA, 2000.

16. National Committee for Clinical Laboratory Standards. Methods for dilution antimicrobial susceptibility tests for bacteria that grow aerobically: approved standard, M7-A55th. 5th ed. Wayne, PA, 2000.

17. Pallares R, Liñares $\mathrm{J}$, Vadillo $\mathrm{M}$, et al. Resistance to penicillin and cephalosporin and mortality from severe pneumococcal pneumonia in Barcelona, Spain. N Engl J Med 1995;333:474-480.
18. Bergan T. Pharmacokinetics of the fluoroquinolones. In: Andriole VT, ed. The quinolones. 2nd ed. San Diego: Academic Press 1998;143182.

19. Cunha BA, Shea KW. Emergence of antimicrobial resistance in community-acquired pulmonary pathogens. Semin Respir Infect 1998;13: 43-353.

20. Jacobs MR, Bajaksouzian S, Zilles A, Lin G, Pankuch, Appelbaum P Susceptibilities of Streptococcus pneumoniae and Haemophilus influenzae to 10 oral antimicrobial agents based on pharmacodynamic parameters: 1997 US Surveillance Study. Antimicrob Agents Chemother 1999:43:1901-1908.

21. Thornsberry C, Ogilvie P, Kahn J, Mauriz Y, Laboratory Investigator Group. Surveillance of antimicrobial resistance in Streptococcus pneumoniae, Haemophilus influenzae, and Moraxella catarrhalis in the United States in 1996-1997 respiratory season. Diagn Microbiol Infect Dis 1997;29:249-257.

22. Pontani D, Washton H, Bouchillon S, Johnson J. Susceptibility of European respiratory tract isolates to trovafloxacin, ciprofloxacin, clarithromycin, azithromycin and ampicillin. Eur $\mathrm{J}$ Clin Microbiol Infect Dis 1998; 17:413-419.

23. Butler DL, Gagnon RC, Miller LA, Poupard JA, Felmingham D, Gruneberg RN. Differences between the activity of penicillin, amoxycillin, and co-amoxyclav against 5,252 Streptococcus pneumoniae isolates tested in the Alexander Project 1992-1996. J Antimicrobial Chemother 1999;43:777-782

24. Tomasz A, Corso A. Members of the PAHO/Rockefeller University Workshop, Severina EP, Achániz-Aviles G, Brandileone MCC, Camou T, Castañeda E, Figueroa O, Rossi A, DiFabio JL. Molecular epidemiologic characterization of penicillin-resistant Streptococcus pneumoniae recovered in six Latin-American countries: an overview. Microbial Drug Resist 1998;4:195-207.

25. Acar JF. Resistance patterns of Haemophilus influenzae. J Chemother 1999;11(Suppl 1):44-50.

26. Ball P. Therapy for pneumococcal infection at the millennium: doubts and certainties. Am J Med 1999;107:77S-85S.

27. Doern GV, Jones RN, Pfaller MA, Kugler K. Haemophilus influenzae and Moraxella catarrhalis from patients with community-acquired respiratory tract infections: antimicrobial susceptibility patterns from the SENTRY Antimicrobial Surveillance Program (United States and Canada, 1997). Antimicrob Agents Chemother 1999;43:385-389.

28. Miranda Novaes MG, Solorzano Santos F, Guiscafre Gallardo H, et al. Streptococcus pneumoniae: low frequency of penicillin resistance and high resistance to trimethoprim-sulfamethoxazole in nasopharyngeal isolates from children in a rural area in Mexico. Arch Med Res 1997 28:559-563.

29. Jenigan DB, Cetran MS, Breiman RF. Minimizing the impact of drugresistant Streptococcus pneumoniae (DRSP). JAMA 1996;275:206209.

30. Brandileone MC, Di Fabio JL, Vieira VSD, Zanella RC, Casagrande ST, Pignatari AC, Tomasz A. Geographic distribution of penicillin resistance of Streptococcus pneumoniae in Brazil: genetic relatedness Microbial Drug Resist 1998;4:209-217.

31. Thorburn CE, Knott SJ, Edwards DI. In vitro activities of oral betalactams at concentrations achieved in humans against penicillin-susceptible and resistant pneumococci and potential to select resistance. Antimicrob Agents Chemother 1998;42:1973-1979.

32. Dagan R, Abramson O, Leibovitz E, et al. Goshen S, Greenberg D, Yagupsky P, Leiberman A, Fliss DM. Impaired bacteriologic response to oral cephalosporins in acute otitis media caused by pneumococci with intermediate resistance to penicillin. Pediatr Infect Dis J 1996 15:980-985 
33. Jones RN, Johnson DM, Erwin ME, et al. Comparative antimicrobial activity of gatifloxacin tested against Streptococcus spp. including quality control guidelines and Etest method validation. Diagn Microbiol Infect Dis 1999;34:91-98.

34. Perry CM, Berman JAB, Lamb HM. Gatifloxacin. Drugs 1999; 58 683-696.

35. Milatovic D, Schmitz F, Brisse S, Verhoef J, Fluit C. In vitro activity of sitafloxacin (DU-6859a) and six other fluoroquinolones against 8,796 clinical bacterial isolates. Antimicrob Agents Chemother 2000;44: $1102-1107$.

36. Balfour JAB, Wiseman LR. Moxifloxacin. Drugs 1999;57:363-373.
37. Del'Alamo L, Sampaio J, Miranda EA, Sader HS. Comparative in vitro activities of moxifloxacin (BAY 12-8039) and other antimicrobial agents against respiratory tract pathogens in Brazil. Braz J Infect Dis 1999;3:215-219.

38. Jones RN, Johnson DM, Erwin ME, et al. Comparative antimicrobial activity of gatifloxacin tested against Streptococcus spp. including quality control guidelines and Etest method validation. Diagn Microbiol Infect Dis 1999;34:91-98.

39. Lipsky BA, Baker CA. Fluoroquinolones toxicity profiles: a review focusing on newer agents. Clin Infect Dis 1999;28:352-364.

40. Anonymous. Gatifloxacin and moxifloxacin: two new fluoroquinolones. Med Lett Drugs Ther 2000;42:15-17. 